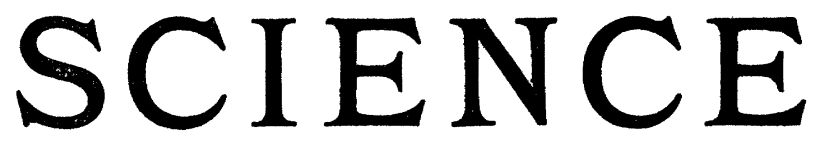

FrIDAY, JULY 17, 1914

CONTENTS

The Principtes of the Theory of Mutation: Professor Hugo de VRIES .............

The Problem of Lighting in its Relation to the Efficiency of the Eye: Dr. C. E. FerreE.

Carl Fuchs: Frank E. Blaisdeld, SR. .....

Estimates of Population ................

Scientific Notes and News ..............

University and Educational News ..........

Discussion and Correspondence:-

Lightning Flashes: Dr. Clleveland ABBe. A New Form of Collecting Pipette: ARTHUR M. BANta. Is Melanism due to Food? WM.

T. $\operatorname{Cox} \ldots \ldots \ldots \ldots \ldots \ldots . . . . . . . .$.

\section{Scientific Books:-}

Iddings on Igneous Rocks: DR. WHITMAN Cross. Allen's Commercial Organic Analysis: Professor Lafayette B. Mendel. Fantham and Porter on Minute Animal Parasites: Professor GARY N. CaLKins.

Special Artictes:-

Direct Proof through Non-disjunction that the Sex-linked Genes of Drosophila are Borne by the $X$-chromosome: CALviN $\mathrm{B}$. BRIDGES. Hot-water Treatment for Cotton Anthracnose: H. W. BARRE AND W. B. Aull $\ldots \ldots \ldots \ldots \ldots \ldots \ldots \ldots \ldots . .107$

The American Chemical Society. IV: DR. Charles L. Parsons ................. 110

MSS. intended for publication and books, etc., intended for review should be sent to Professor J. McKeen Cattell, Garrisonon-Hudson, N. Y.

\section{THE PRINCIPLES OF THE THEORY OF MUTATION 1}

UNITY of internal structure combined with a great diversity of external forms is read my books. It is obviously erroneous and therefore may well be called a myth. Logically and historically the desirability of those experiments has been derived from the theory, as will be seen in the text. Jeffrey bases his arguments upon the well-known researches of Geerts concerning the partial sterility of many of the members of the natural family of the Onagracear. Geerts found that in almost all the genera of this family, including all their species as far as investigated, the ovules are for one half in a rudimentary condition, which excludes the possibility of their being fertilized, whilst about one half of the pollen grains is sterile. This double character has therefore persisted during the pedigree-evolution of almost this whole family. In contradiction with Geerts, Jeffrey considers it to be an indication of a hybrid condition. If this were true, almost the whole natural family of the Onagracece would have evolved in a hybrid condition and Enothera Lamarckiana would follow the rule. It remains doubtful, however, how this hypothesis could explain the high degree of mutability of $O$. Lamarckiana, since the majority of the supposed hybria species do not show signs of such a condition. 
It has been the work of Darwin to accumulate a large number of facts and arguments, borrowed from the most diverse parts of the physical and biological sciences, and to combine the main results of the study of nature in general in order to find a conclusive proof of the idea of Lamarck. Common descent is now acknowledged as the natural cause of the unity of organization. Successive slow modifications have produced the great diversity of forms and the diverging lines of evolution which have gradually led to the highest degrees of differentiation.

But his broad views and comprehensive considerations did not suffice to afford the desired proof. Comparative anatomy and systematical studies, the knowledge of the laws of the geographical distribution of animals and plants and of their gradual development during the geological epochs, could only outline the broad features of the theory. Evidently its basis must be sought in the study of the process by which one species is produced from another. Which is the nature and which are the causes of this process? Which are the elementary changes which, by numerous repetitions and combinations, have produced the main evolutionary lines of the animal and vegetable kingdom?

In order to answer these questions, Darwin studied the experience of the breeders. The improvement of domestic animals was well known at his time, the cultivated races of flowers and vegetables, of cereals and sugar-beets clearly and widely surpassed the same species in nature.

The method of breeders is based on the principles laid down about the middle of the last century (1840) by P. P. A. Lévêque de Vilmorin, the father of the celebrated founder of the culture of sugar-beets. He had observed the high degree of variability of cultivated plants and discovered that by means of a choice of the best samples and by their isolation highly improved varieties may be produced. His son has applied this principle to the sugar-beets, one of the most variable of all cultivated forms, and succeeded in increasing the amount of sugar from 7 to 14 per cent. This improvement soon became the basis of a large sugarindustry in many countries of Europe. From that time isolation and selection have become the watchwords of a big new industry, which soon produced the most unexpected results in almost all parts of agricultural practise.

Darwin transplanted this principle of practise into pure science. He studied the variability of species in the wild condition and found it as widely spread and as rich in its features as in cultivated forms. He saw that very many species are distributed in nature in such a way as to constitute numbers of isolated colonies, sufficiently distant from one another to exclude the possibility of intercrossing. He discovered the great factor which replaces artificial selection in nature and called it by the name of natural selection. It is the unceasing struggle for existence and the victory of the most endowed individuals. In nature, every plant produces more seeds than can develop into new plants, owing to lack of space. Only those which are most fit for the surrounding conditions will survive, whilst the remainder are condemned to disappear. In this manner the struggle for life leads to a selection, which will be repeated in every generation, and a whole colony may gradually change by this means until at the end the characters are sufficiently different from the original ones to constitute a new variety or even an elementary species.

Natural selection in the struggle for life has now become the main principle of organic evolution. Since species obey in 
the wild condition the same laws as under cultivation, the principles of their improvement must be the same everywhere.

Darwin applied this principle to geological evolution also. Lyell had shown that the laws of nature have always been the same from the very beginning. Therefore natural selection must have been active from the first time of the existence of life on earth and have produced the main lines of differentiation as well as the first traces of all those groups, which are now recognized as families and genera. It is my conviction that the success of Darwin in this line of ideas has been as complete as possible. He succeeded in convincing his contemporaries of the essential analogy between artificial and natural selection.

But, on the other hand, it must be conceded that the practise of breeders was not as simple as it seemed to be. No thorough study of the phenomena of variability had been made, and it was simply assumed that the diversity of forms within the cultivated races was due to one cause only. This was indicated by the well-known expression that no two individuals of a race are exactly alike. All specimens differ from one another in their industrial qualities as well as in their botanical characters. These qualities and characters are inheritable and the offspring of a selected individual will vary, according to Vilmorin, around an average lying between the type of the original species and that of the chosen individual. By this means the range of variability will be extended in the desired direction, and this may be repeated during a number of years, until the industrial value of the new race clearly surpasses that of the old one.

Evidently, it was said, natural selection must work in the same way. But the question remains whether this will really lead to new species, or only to local and temporary adaptations.
The answer to this question has been given by the newest discoveries of agricultural practise itself. Hjalmar Nilsson, the director of the celebrated experimental station of Svalöf in Sweden, discovered that variability among cultivated plants is not a single phenomenon, but contains at least two widely contrasted features.

He found that, apart from fluctuating variability, every cultivated species is a mixture of elementary types. A field of a cereal is only apparently uniform, and a closer investigation soon reveals numerous differences in the height of the stems, in the time of flowering, in the size and almost all other qualities of the ears, in resistance to diseases and especially in the industrial value of the grains. Moreover, he found that all these qualities are strictly inheritable. Nilsson took the grains of a single ear and found that all the individuals issuing from them are strictly alike and carefully repeat the characters of their mother. From such a chosen ear one may derive by repeated sowings grain enough to sow a whole field, and this will show an almost complete and very striking uni. formity. Therefore our ordinary species and varieties of cultivated plants are in reality mixtures of a smaller or larger number of different races, which grow together, but are, as a matter of fact, independent of one another. These races themselves are almost invariable, but their mixture in the field produces upon us the impression of a great variability.

What is the significance of this discov-. ery for the explanation of artificial selection? Evidently this will tend to isolate the better races of the mixture and to exclude those of average or low value. Two methods may be followed. Either the breeder collects a handful of ears chosen with the utmost care from all parts of his field and secures a lot of grains large 
enough to sow a parcel of a moderate extent. Or he limits his choice to one ear only, which will take him one year more to obtain the necessary quantity of grains. The first method is the one which is still commonly followed, the second was introduced some twenty years ago by Nilsson.

The real nature of the first method may be explained by means of the careful studies of Rimpau, who applied it for the improvement of his rye. The group of ears of the first choice will evidently be itself a mixture, although of a lesser number of types. In choosing year after year a handful of the best ears Rimpau must gradually have purified this mixture, until after twenty years he succeeded completely in isolating the very best one of them. From this time his race must have been pure and constant, no further selection being possible. Using the method of Nilsson the same result may be reached by a single choice, and therefore in one year. The new race is produced by a jump and not by the slow and gradual improvement by small and almost invisible steps, which was assumed by Rimpau and Darwin.

From these discoveries the question arises, whether natural selection also proceeds by jumps and leaps, and not, as was commonly assumed, by imperceptible steps. The answer may be deduced from the observations of Jordan and others on the existence of elementary species in nature. Almost every wild species consists of some of them, and in special cases their number increases so as to embrace dozens or even hundreds of sharply distinguished types. Sometimes these are found in widely distant stations; at other times, however, they are growing in mixtures. Natural selection will, of course, under changed conditions, simply multiply one or two of the types to the exclusion of the others. As a whole, the species will make progress in the desired direction, but in reality there will be no change of forms.

From all these and many other considerations it follows that the basis, which the practise of artificial selection seemed to afford to the theory of natural selection, is a fallacious one, and that the idea of evolution by means of slow and almost imperceptible steps must therefore be abandoned. But if this is conceded, how are species really produced in nature?

The theory of mutations answers that species are produced by means of jumps and leaps, exactly in the same way as varieties in horticulture. Varieties are only beginning species, says Darwin, and the same laws must govern the origin of both of them. Now, in horticulture, it is well known that varieties usually arise at once. In a field of a species with blue or red flowers some day an individual with white flower is seen. Ordinarily it is only one, and it is not surrounded by transitions or by flowers of intermediate colors. Sometimes there may be two or three, but then their flowers are of the same degree of whiteness. One seed of the species has been transformed into a variety, and this is its whole origin. A single season suffices to produce the effect, no slow and gradual improvement being required. Moreover, the seeds of the first individual, if fertilized and saved separately, will reproduce the variety wholly pure. The same rule prevails for large groups of other cases; everywhere varieties arise by jumps, requiring only one year for their arrival.

The same rule also holds good in nature. But in order to show this, direct experiments are required. For this object I have cultivated a large number of wild species in my experiment garden, trying to see them produce varieties and to be enabled thereby to study the laws of this process. Let me adduce two instances, the origin of the 
peloriated toadflax and that of the double variety of the corn marigold. These varieties appeared in my cultures all of a sudden, after a number of years, the one in about half a dozen of individuals in successive generations, the other in a single instance. The ordinary toadflax has only one spur on its flowers and remained so in hundreds of individuals until a single specimen bore five spurs on every one of its flowers. The corn marigold had normal flower heads until 1899 when one individual produced some slight signs of duplication. Next year all its descendants bore double flowers and the race showed itself constant from the very beginning.

Thus, the production of varieties by leaps and jumps may be considered as a wellproved fact in horticulture and in a state of nature. It is a firm basis for a new theory, and we have only to transport the principle from the varieties to the origin of elementary species. Recognized for species, the theory will obviously be true for genera and families also, and explain the evolution of all organic beings in all the different lines of the genealogical tree.

The idea of the origin of species by leaps and jumps has the great advantage of answering in an unexpected and decisive way the numerous and in part very grave objections which have been brought forward against the theory of Darwin. To my mind, this is one of the best arguments in its favor. It releases the theory of evolution from the serious difficulties which its adversaries have never ceased to urge against it. Therefore it seems useful to give a brief survey of them now.

The oldest and most serious objection is based on the obvious uselessness of new characters during the first stages of their evolution, if this is supposed to be invisibly slow. Imperceptible odors can not guide insects in their visits to flowers and assure to these a sufficient advantage in the struggle for life. Adaptations for the capturing of insects by plants would be of no value in a primary and imperfect condition and therefore can not be evolved by the action of natural selection. Imperfect instincts would be rather obnoxious, according to Wasmann, and thus would be liable to be destroyed instead of increased by this action. So it is in many other cases. Beginning characters would always be too insignificant to be of any value in the struggle for life. Evidently the principle of leaps and jumps at once relieves us of the necessity of this hypothesis. It does not admit a gradual appearance of characters, but assumes these to appear at once in the full display of their development, and without the aid of natural selection.

The same holds good for useless characters. The theory of Darwin can not explain them. According to him, every quality is developed exactly through its utility, and useless properties should be eliminated from the very beginning by the struggle for life. But it is now generally recognized that many beautiful differentiations are in reality no adaptations at all, and that their usefulness is at least very doubtful. This, for instance, is the case of heterostyly and of the likeness of the flowers of some orchids to insects. The theory of mutations has no difficulty with useless and even with slightly prejudicial characters. Arising by a sudden jump, they may keep their place, provided only that they are not in such a degree hurtful as to prevent a normal development of the individuals.

A third objection has been derived from the studies of the celebrated anthropologist Quetelet, who discovered the general law of fluctuating variability. $\mathrm{He}$ introduced the principle of studying every quality for itself and of comparing the different 
degrees of its development in a large number of individuals. He found by this means that characters simply follow the laws of probability. They vary around an average condition in two directions, of increase and decrease, but precisely thereby this variability excludes the production of a new character. Darwin tried to derive the one from the other, whilst the theory of mutations recognizes the almost diametrically opposed nature of the two phenomena.

A last objection has been brought forward by the study of the age of the earth. Physicists as well as astronomers have refused to accept the theory of slow evolu. tion as the time required by Darwin in connection with his ideas, seemed by far too long. A man's life would not suffice to see the changes, which, after him, would be necessary to produce a single step in the line of evolution. The differentiation of a flower or of a seed would require millions of years if it went on so slowly, and the development of the whole organism of a plant, and still more so that of the higher animals, would obviously require a vastly larger amount of time. Darwin has calculated the necessary time for the evolution of the whole animal and plant kingdom on the assumption of slow and almost imperceptible changes, and estimated it to be at least equal to some thousands of millions of years.

But our globe can not be as old as that. There is quite a large number of arguments which allow us to estimate the age of the earth with a sufficient degree of accuracy, and they all point, unanimously, to a period of only some twenty or forty millions of years. This number is evidently far too small for the explanation given by Darwin and in consequence thereof it has always been considered as one of the most decisive arguments against the theory of slow and gradual evolution.
In order to estimate the age of the earth different phenomena may be used. First the separation of the moon, secondly the solidification of the earth's crust, then the condensation of the aqueous vapor and the formation of oceans. The quantity of salt dissolved in these oceans and the thickness of the geological layers, especially those of a calcareous nature, afford further arguments.

According to George Darwin the moon was separated from our globe about 56 millions of years ago. The age of the solid crust has been calculated by Lord Kelvin from the increase of the temperature in deep mines. In some regions the temperature is seen to increase about one degree for every fifty meters; in others, however, one degree for a hundred meters. On the average the considerations of Lord Kelvin gave an age of twenty to forty millions of years for the solid crust of the earth.

The quantity of salt obviously increases in the oceans on account of the salt added by the rivers and of the evaporation of the water. The total quantity of this salt has been calculated and the quantities of the yearly supply of water are known for all the larger streams, as well as their percentage of salt. From these data we may calculate the annual increase of salt in the oceans and find how many years would be required for our present rivers to accumulate all the salt now found in the seas. According to Joly, about ninety millions of years would be necessary. But obviously the rivers must exhaust the grounds which they drain, and formerly these must therefore have been much richer in salts. This consideration must lead us to diminish the number of years required in a very sensible manner.

The age of the geological strata has been deduced from their thickness and the velocity of the process of sedimentation. 
Sollas estimates the total thickness at about 80 kilometers and the average rate of deposition of the layers at $30 \mathrm{~cm}$. per century. From these numbers we may find an age of 26 millions of years for the collective deposition of all the geological layers. Calcareous rocks have been built by organisms and mainly by corals and molluscs. These have made use of the lime added to the sea by the rivers. Dubois has calculated on the one hand the whole thickness of these rocks and on the other the yearly supply of lime from the rivers. He concludes that $36-45$ millions of years would be required to produce the whole of this system.

All these data have been subjected to a criticism by Sollas and compared with one another. Obviously the highest estimates are only limits, and in considering this, Sollas arrives at the general average of about 20-40 millions of years. He points out that the epochs which have served as starting points are not very far distant from one another, considered in a geological way, and that therefore they may be taken together to delineate the duration of organic life on this earth.

As we have seen, this duration is by far too short to allow the slow and gradual development of life supposed by Darwin. It necessitates a very substantial abbreviation of this process and thus affords one of the best supports of the theory of mutations.

Thus we see that this theory is based on almost all the branches of natural science. All of them join in the assertion that the hypothesis of slow and almost invisible changes is too improbable to be accepted and is even in open contradiction to some of the best results of other sciences. The theory of an evolution by leaps and jumps evades all these objections and thereby releases the theory of Darwin from its separate position.
But it is doing more than this. By rejecting the hypothesis of invisible changes it leads us to search for the visible alterations, which it assumes to be the leaps and jumps by which animal and vegetable species are being produced. If the transformation of one species into another is a visible process, it must evidently be sought for and be brought to light in order to study its laws, and to derive from this study an experimental proof for the theory of evolution.

However, it is hardly probable that these jumps are numerous in nature as it now surrounds us. On the contrary, they must rather be rare, since nobody had seen them until now in the field. Therefore I have sought for a plant which would produce more of such mutations than other plants. I have studied over a hundred species, investigating their progeny, and among them one has answered my hopes. This is the evening primrose of Lamarck, which chances to bear the name of the founder of the theory of evolution which it is prepared to support. It is a species which grew wild in the territory of the United States, where it has been collected by the well-known traveler and botanist Michaux, and whence Lamarck derived the authentic specimen for his description. Since that time it has spread in Europe and is now found especially in England, Belgium and Holland in a number of localities, some of which consist of many thousands of individuals. In more than one of these localities it has been observed to produce mutations, especially in a field near Hilversum in Holland, whence I have obtained the individuals and seeds which have served as the starting points of my cultures.

In these cultures the species is seen to be very pure and uniform in the large majority of its offspring, but to produce on an average one or two aberrant forms in every 
hundred of its seedlings. The differences are easily seen even in young plants and are mostly large enough to constitute new races. The more common ones of these races are produced repeatedly, from the seed from the wild plants as well as in the pure lines of my cultures. It is obviously a constant and inheritable condition which is the cause of these numerous and repeated jumps.

These jumps at once constitute constant and ordinarily uniform races, which differ from the original type either by regressive characters or in a progressive way. By means of isolation and artificial fecundation these races are easily kept pure during their succeeding generations.

I shall not insist here upon their special characters. The most frequent form is that of the dwarfs, Enothera nanella, and the rarest is the giant, or 0 . gigas, which has a double number of chromosomes in its nuclei (28 instead of 14) and by this mark and its behavior in crossing proves to be a progressive mutation. Other new types which are produced yearly are $O$. rubrinervis, $O$. oblonga and $O$. albida. O. lata is a female form, producing only sterile pollen in its anthers and $O$. scintillans is in a splitting condition, returning every year in a greater or less number of individuals to the original type from which it started. Besides these there are a large number of mutations of minor importance, many of which have not even been described up to the present time.

Thus we see that the experiments provide us with a direct proof for the theory of evolution. They constitute an essential support of the views of Darwin, and moreover they relieve them of the many objections we have quoted and bring them into harmony with the results of the other natural sciences.

But, besides this, they show us the way into a vast new domain of investigation and afford the material for a study of the internal and external causes which determine the production of new species, at least in those cases in which, as in the primroses, mutations are relatively abundant. From these we may confidently hope to come some day to the study of those rarer mutations on which the differentiation of the main lines of organic evolution seem to have depended. Hugo de VRIES

UNIVERSity of AMsterdaM

\section{THE PROBLEM OF LIGHTING IN ITS RE- LATION TO THE EFFICIENCY OF $T H E$ EYE1}

UP to the present time the work on the problem of lighting has been confined almost entirely to the source of light. The goal of the lighting engineer has been to get the maximum output of light for a given expenditure of energy. Until recent years little attention has been given to the problem in its relation to the eye. It is the purpose of this paper to outline in a general way some of the more important features of this phase of the subject, and to give some of the results of work that is now being done on the problems that these features present.

Confronting the problem of the effect of lighting systems on the eye, it is obvious that the first step towards systematic work is to obtain some means of making a definite estimate of this effect. The prominent effects of bad lighting systems are loss of efficiency, temporary and progressive, and eye discomfort. Three classes of effect may, however, be investigated: (1) the effect on the general level or scale of efficiency for the fresh eye; (2) loss of efficiency as the result of a period of work; and (3) the tendency to produce discomfort. Of these three classes of effect the last two are obviously the more important, for the best lighting system is not the one that gives us the maximum acuity of vision

1 This paper, with some changes, was read before the American Philosophical Society of Philadelphia, April 4, 1913. 